\title{
Простейшие комбинаторные типы выпуклых полиэдров с группами симметрии средней сингонии и икосаэдрическими группами симметрии -3-5m и 235
}

\section{Степенщиков Д.Г. ${ }^{1}$, Войтеховский Ю.Л. ${ }^{2}$}

${ }^{1}$ Геологический институт КНЦ РАH, stepen@geoksc.apatity.ru

${ }^{2}$ Санкт-Петербургский горный университет, Voytekhovskiy_Yul@pers.spmi.ru

Аннотация. Для каждой группы симметрии средней сингонии найдена общая форма комбинаторных типов выпуклых полиэдров с минимальным числом граней. Найдены комбинаторные типы выпуклых полиэдров с минимальным числом граней для икосаэдрических групп симметрии -3-5m и 235 .

Ключевые слова. Комбинаторный тип, некристаллографическая группа симметрии, средняя категория симметрии, икосаэдрическая симметрия, выпуклый полиэдр.

\section{The simplest combinatorial types of the convex polyhedra with symmetry groups of the middle category and icosahedral symmetry groups $-3-5 m$ and 235}

\author{
Stepenshchikov D.G. ${ }^{1}$, Voytekhovsky Yu.L. ${ }^{2}$ \\ ${ }^{1}$ Geological Institute KSC RAS, stepen@geoksc.apatity.ru \\ ${ }^{2}$ Saint Petersburg Mining University,Voytekhovskiy_Yul@pers.spmi.ru
}

Abstract. For each symmetry group of the middle category the general shape of combinatorial types of the convex polyhedra with minimum number of faces is found. For icosahedral symmetry groups $-3-5 m$ and 235 combinatorial types of the convex polyhedra with minimum number of faces are found.

Key words. Combinatorial type, non-crystallographic symmetry group, middle category of symmetry, icosahedral symmetry, convex polyhedron.

В статье (Войтеховский, Степенщиков, 2020) показан метод доказательства существования простейших комбинаторных типов на примере групп симметрии $m-3$ и 432. Далее рассмотрен общий случай для групп симметрии средней категории. Найдены простейшие комбинаторные типы для недостающих 6 кристаллографических и бесконечного числа некристаллографических групп симметрии с главной осью любого порядка.

На рисунке 1 дан общий вид (т.е. при произвольном порядке $\mathrm{n}$ главной оси) ранее найденных простейших комбинаторных типов для групп симметрии средней категории. Рассмотрим для каж-

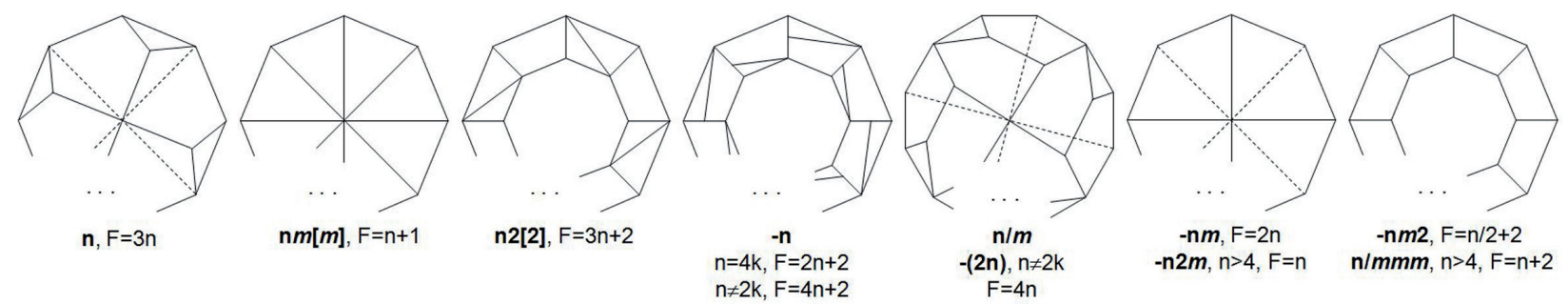

Рис. 1. Общий вид простейших комбинаторных типов для групп симметрии средней категории с числом граней $\mathrm{F}$ и порядком главной оси $\mathrm{n}$. Квадратные скобки означают, что при нечетности $\mathrm{n}$ символ внутри них не пишется в обозначении группы симметрии. Для $-\mathrm{n} 2 m \mathrm{n} n / m m m \mathrm{n}>4$ в связи с тем, что при $\mathrm{n}=4$ указанные простейшие комбинаторные типы имеют более высокую симметрию тетраэдра и куба, соответственно.

Fig. 1. The general shapes of the simplest combinatorial types for symmetry groups of the middle category with $\mathrm{F}$ number of faces and n-fold main axis. Square brackets means the symbol within ones is absent in symmetry group notation for odd $n$. For $-n 2 m$ and $n / m m m n>4$ because provided $n=4$ the simplest combinatorial types has higher tetrahedral and cubic symmetry, respectively. 
дой группы симметрии все возможные комбинации простых форм, порождающих полиэдры с числом граней, меньшим, чем F.

Заметим, что для групп симметрии $\mathrm{n} m[m],-\mathrm{n} m,-\mathrm{n} 2 m,-\mathrm{n} m 2$ и $\mathrm{n} / m m m$ таких комбинаций нет. Так, для группы симметрии $\mathrm{n} / \mathrm{mmm}$ можно исключить из рассмотрения $2 \mathrm{n}$ - и ди-n-гональные призмы; n-, 2n- и ди-n-гональные дипирамиды с числом граней, большим, чем n+2. Оставшиеся n-гональная призма и пинакоид порождают единственный полиэдр с простейшим комбинаторным типом, указанным на рисунке 1 .

Таблица 1. Все комбинации простых форм группы симметрии n, порождающие полиэдры с числом граней, меньшим, чем 3n.

Table 1. All combinations of the simple forms for $\mathrm{n}$ symmetry group, producing polyhedra with number of faces less than $3 \mathrm{n}$.

\begin{tabular}{|c|c|c|c|c|}
\hline № & моноэдры & $\begin{array}{c}\text { n-гональные } \\
\text { призмы }\end{array}$ & $\begin{array}{c}\text { n-гональные } \\
\text { пирамиды }\end{array}$ & $\begin{array}{c}\text { число } \\
\text { граней }\end{array}$ \\
\hline 1 & & & 2 & $2 \mathrm{n}$ \\
\hline 2 & 1 & & 1 & $\mathrm{n}+1$ \\
\hline 3 & 1 & & 2 & $2 \mathrm{n}+1$ \\
\hline 4 & 1 & 1 & 1 & $2 \mathrm{n}+1$ \\
\hline 5 & 2 & & 1 & $\mathrm{n}+2$ \\
\hline 6 & 2 & & 2 & $2 \mathrm{n}+2$ \\
\hline 7 & 2 & 1 & & $\mathrm{n}+2$ \\
\hline 8 & 2 & 1 & 1 & $2 \mathrm{n}+2$ \\
\hline 9 & 2 & 2 & & $2 \mathrm{n}+2$ \\
\hline
\end{tabular}

Для группы симметрии n все 9 возможных комбинаций простых форм с числом граней меньше $3 n+2$ даны в табл. 1. Комбинации призм с моноэдрами (№№ 7,9) и пирамиды с моноэдрами (№№ 2, 5) порождают полиэдры с вертикальными плоскостями симметрии, т.е. их комбинаторные

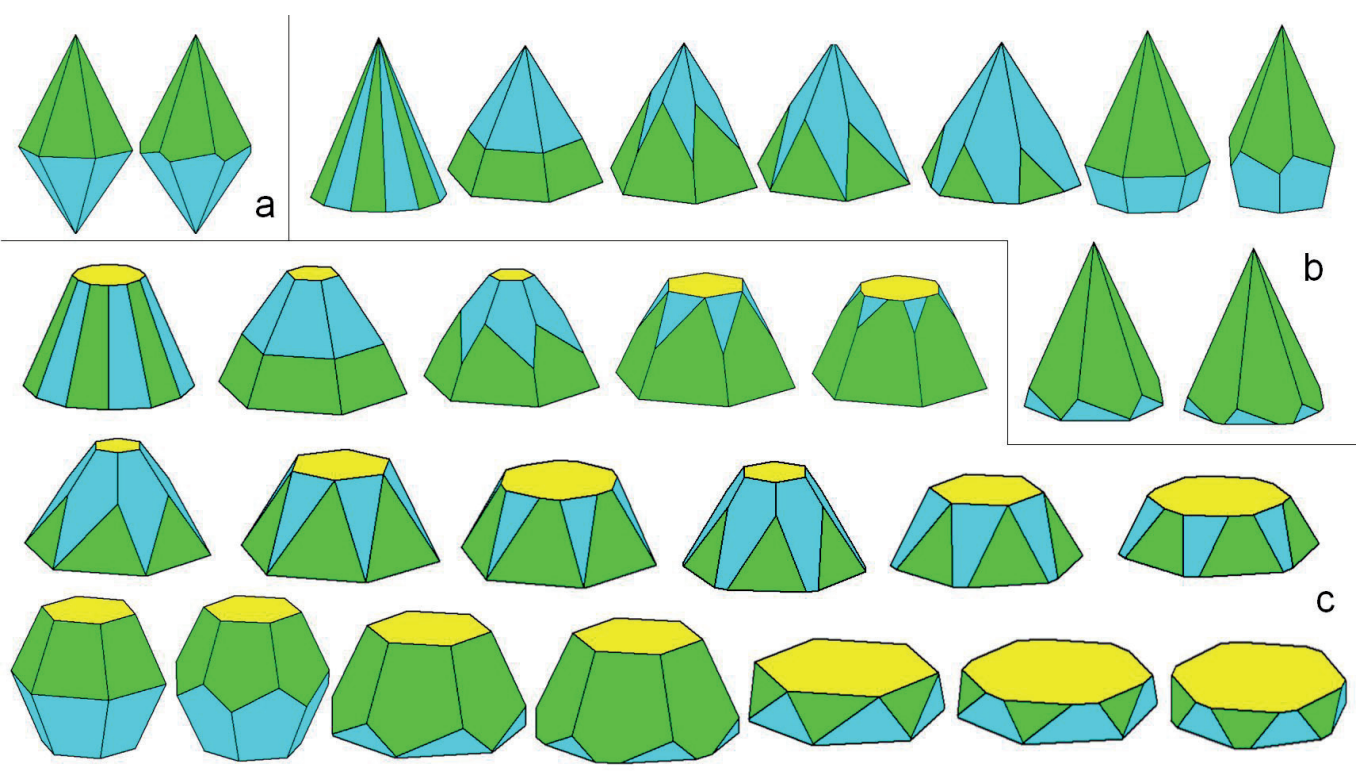

Рис. 2. а - все комбинации двух n-гональных пирамид, b - двух n-гональных пирамид и моноэдра, с - двух n-гональных пирамид и двух моноэдров. Здесь и далее на рисунках даны изображения полиэдров с конкретным значением n (здесь 6). Но для иллюстрации периодичности строения поверхности при любом n это не существенно.

Fig. 2. a - all combinations of two n-gonal pyramids, b - of two n-gonal pyramids and monohedron, c - of two n-gonal pyramids and two monohedra. Hereinafter polyhedra with definite $\mathrm{n}$ (6 in this case) are drawn. But for illustration of periodic-built surface for any $\mathrm{n}$ it is not significant. 
типы заведомо имеют более высокую комбинаторную симметрию. Комбинаторно различные полиэдры для оставшихся комбинаций двух пирамид (№ 1), двух пирамид с моноэдром (№ 3), двух пирамид с двумя моноэдрами (№ 6), пирамиды с призмой и моноэдром (№ 4), пирамиды с призмой и двумя моноэдрами (№ 8) показаны на рисунке 2. В последних двух комбинациях призму можно рассматривать как предельный случай пирамиды.

Таблица 2. Все комбинации простых форм группы симметрии n2[2], порождающие полиэдры с числом граней, меньшим, чем $3 n+2$.

Table 2. All combinations of the simple forms for $n 2[2]$ symmetry group, producing polyhedra with number of faces less than $3 n+2$.

\begin{tabular}{|c|c|c|c|c|c|c|c|}
\hline № & пинакоид & $\begin{array}{c}\text { n-гональные } \\
\text { призмы }\end{array}$ & $\begin{array}{c}\text { 2n-гональные } \\
\text { призмы }\end{array}$ & ди-n-гональные & $\begin{array}{l}\text { n-гональные } \\
\text { дипирамиды }\end{array}$ & $\begin{array}{l}\text { n-гональные } \\
\text { трапецоэдры }\end{array}$ & $\begin{array}{l}\text { число } \\
\text { граней }\end{array}$ \\
\hline 1 & & & & & & 1 & $2 n$ \\
\hline 2 & & & & & 1 & & $2 n$ \\
\hline 3 & & 1 & & & & 1 & $3 n$ \\
\hline 4 & & 1 & & & 1 & & $3 n$ \\
\hline 5 & 1 & & & & & 1 & $2 n+2$ \\
\hline 6 & 1 & & & & 1 & & $2 n+2$ \\
\hline 7 & 1 & & & 1 & & & $2 n+2$ \\
\hline 8 & 1 & & 1 & & & & $2 n+2$ \\
\hline 9 & 1 & 1 & & & & & $\mathrm{n}+2$ \\
\hline 10 & 1 & 2 & & & & & $2 n+2$ \\
\hline
\end{tabular}

Для группы симметрии n2[2] все 10 возможных комбинаций простых форм с числом граней меньше 3n+2 даны в таблице 2. Дипирамида (№ 2), комбинации дипирамиды с пинакоидом (№ 6), а также призм с пинакоидом (№o 7-10) порождают полиэдры с вертикальными плоскостями симметрии, т.е. их комбинаторные типы заведомо имеют более высокую симметрию, чем n2[2]. Трапецоэдр (№ 1) в зависимости от четности n имеет комбинаторный тип с симметрией -n $m$ или $-\mathrm{n} 2 m$. Комбинаторно различные полиэдры для оставшихся комбинаций трапецоэдра с призмой (№ 3), дипирамиды с призмой (№ 4) и трапецоэдра с пинакоидом (№ 5) даны на рисунке 3.

Для группы симметрии -n все 5 возможных комбинаций простых форм с числом граней, меньшим, чем $2 \mathrm{n}+2$ при n, кратном 4 , и меньшим, чем $4 \mathrm{n}+2$ при нечетном $\mathrm{n}$ даны в таблице 3 . Трапецоэдры в данной группе обладают плоскостями симметрии, поэтому трапецоэдр (№ 1), его комбина-
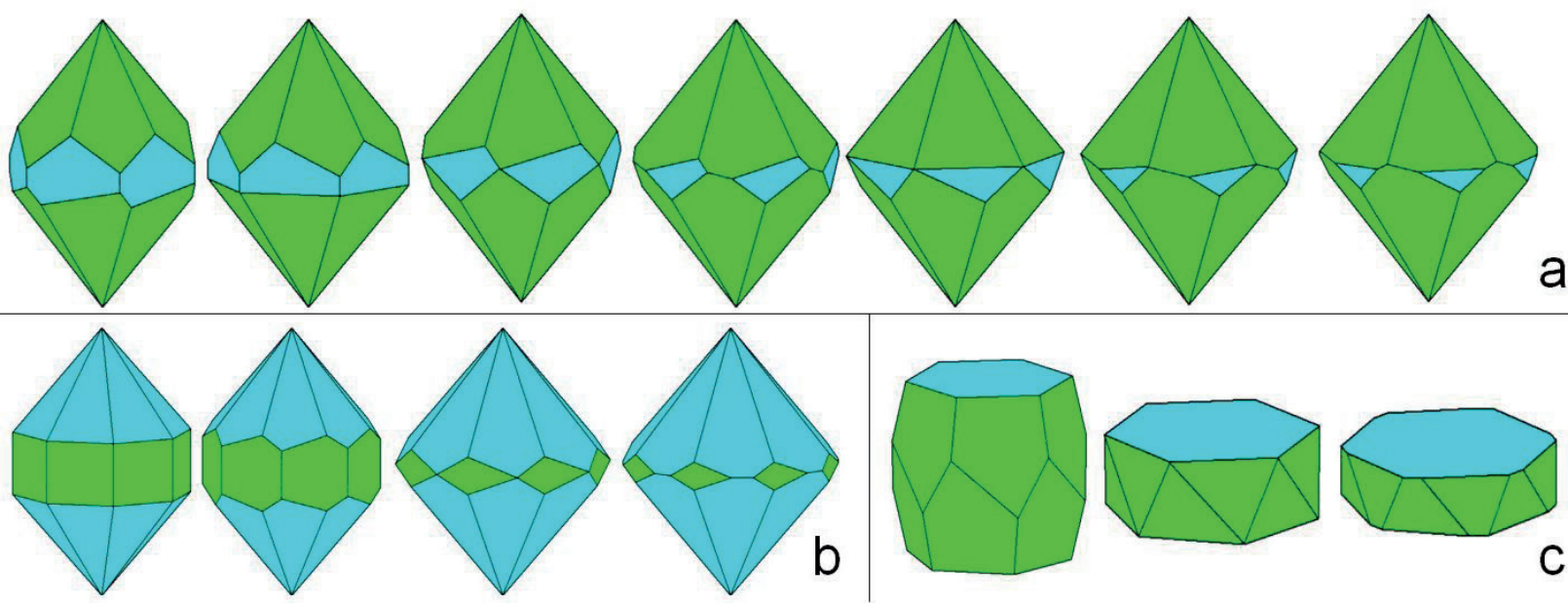

Рис. 3. а - все комбинации n-гональных трапецоэдра и призмы, b - n-гональных дипирамиды и призмы, c - n-гонального трапецоэдра и пинакоида.

Fig. 3. a - all combinations of n-gonal trapezohedron and prizma, $\mathrm{b}$ - of n-gonal bipyramid and prizma, $\mathrm{c}-$ of $\mathrm{n}$-gonal trapezohedron and pinacoid. 
ция с пинакоидом (№ 4), а также комбинация призмы с пинакоидом (№5) порождают полиэдры с вертикальными плоскостями симметрии. Их комбинаторные типы имеют комбинаторную симметрию, заведомо отличающуюся от -n. Комбинаторно различные полиэдры для оставшихся комбинаций двух трапецоэдров (№ 2) и призмы с трапецоэдром (№ 3) даны на рисунке 4.

Таблица 3. Все комбинации простых форм группы симметрии -n, порождающие полиэдры с числом граней, меньшим, чем $2 \mathrm{n}+2$, при $\mathrm{n}$, кратном 4 , и меньшим, чем $4 \mathrm{n}+2$, при нечетном $\mathrm{n}$.

Table 3. All combinations of the simple forms for $-n$ symmetry group, producing polyhedra with number of faces less than $2 n+2$ for $n$ multiple of four and less than $4 n+2$ for odd $n$.

\begin{tabular}{c|c|c|c|c|c|c}
\multirow{2}{*}{ № } & \multirow{2}{*}{ пинакоид } & $\mathrm{n}=4 \mathrm{k}$ & $\begin{array}{c}\mathrm{n} \text {-гональные } \\
\text { призмы }\end{array}$ & $\begin{array}{c}\mathrm{n} / 2 \text {-гональные } \\
\text { трапецоэдры }\end{array}$ & \multicolumn{2}{c}{ число граней } \\
\cline { 3 - 7 } & $\mathrm{n} \neq 2 \mathrm{k}$ & $\begin{array}{c}\text { 2n-гональные } \\
\text { призмы }\end{array}$ & $\begin{array}{c}\mathrm{n} \text {-гональные } \\
\text { трапецоэдры }\end{array}$ & $\mathrm{n}=4 \mathrm{k}$ & $\mathrm{n} \neq 2 \mathrm{k}$ \\
\hline & & & & 1 & $\mathrm{n}$ & $2 \mathrm{n}$ \\
2 & & & & 2 & $2 \mathrm{n}$ & $4 \mathrm{n}$ \\
3 & & & 1 & 1 & $2 \mathrm{n}$ & $4 \mathrm{n}$ \\
4 & 1 & & & 1 & $\mathrm{n}+2$ & $2 \mathrm{n}+2$ \\
5 & 1 & & 1 & & $\mathrm{n}+2$ & $2 \mathrm{n}+2$
\end{tabular}

Для групп симметрии $\mathrm{n} / m$ и -(2n) при нечетном n все 6 возможных комбинаций простых форм с числом граней, меньшим, чем 4n, даны в таблице 4. Дипирамида (№ 1), ее комбинация с пинакоидом (№ 3), а также комбинация призм с пинакоидом (№№ 4, 6) порождают полиэдры с вертикальными плоскостями симметрии, т.е. их комбинаторные типы заведомо имеют более высокую комбинаторную симметрию, отличающуюся от $\mathrm{n} / m$ или $-(2 \mathrm{n})$. Комбинаторно различные полиэдры для оставшихся комбинаций призмы с дипирамидой (№ 2) и призмы с дипирамидой и пинакоидом (№ 5) даны на рисунке 3 b и 5.
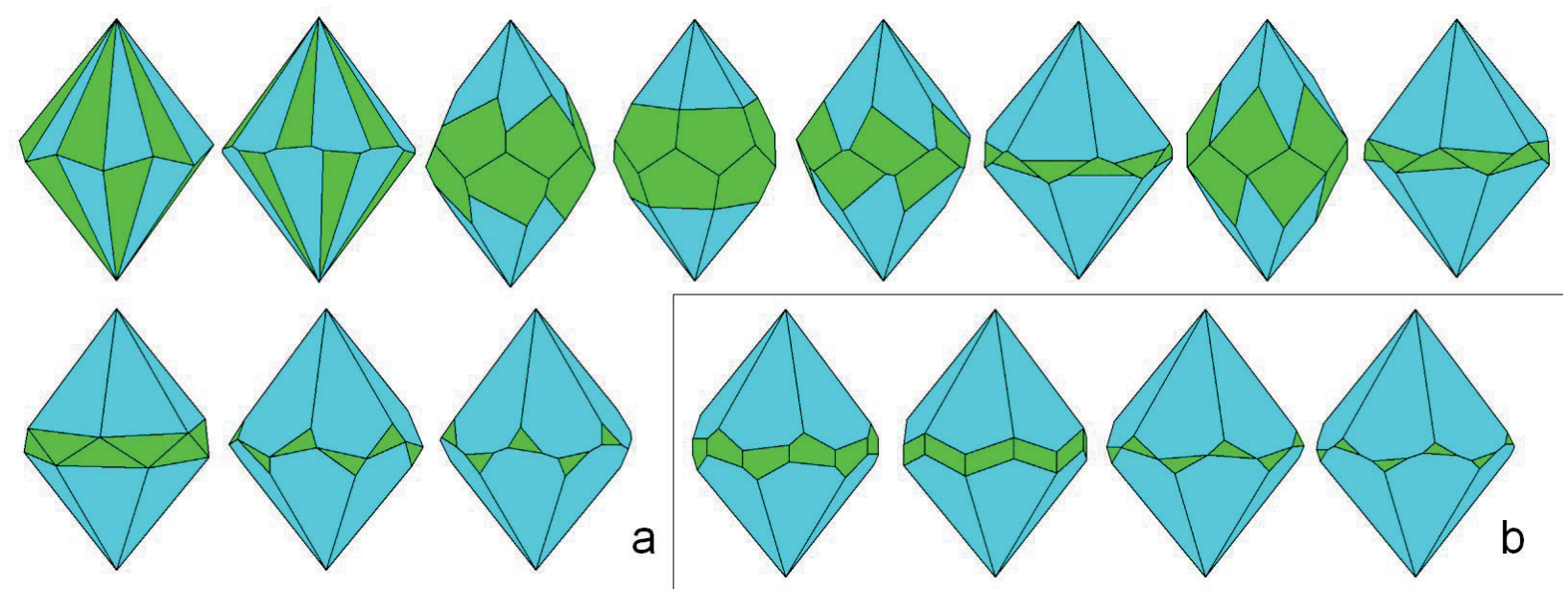

Рис. 4. a-все комбинации двух n-гональных трапецоэдров, $\mathrm{b}$ - 2n-гональной призмы и n-гонального трапецоэдра (или n-гональной призмы и n/2-гонального трапецоэдра).

Fig. 4. a - all combinations of two n-gonal trapezohedrons, $\mathrm{b}$ - of 2n-gonal prizma and n-gonal trapezohedron (or ngonal prizma and $\mathrm{n} / 2$-gonal trapezohedron).

Все полиэдры на рисунке 2-5, за одним исключением, имеют комбинаторные типы с симметрией, обладающей вертикальными плоскостями, не свойственными ни одной из рассматриваемых групп. Исключение составляет второй справа полиэдр на рисунке 3 a, чей комбинаторный тип имеет симметрию $\mathrm{n}$, отличную от $\mathrm{n} 2[2]$. Таким образом, все эти полиэдры имеют комбинаторные типы, симметрии которых отличаются от рассматриваемых групп симметрии. Значит, указанные на рисунке 1. комбинаторные типы - простейшие. 

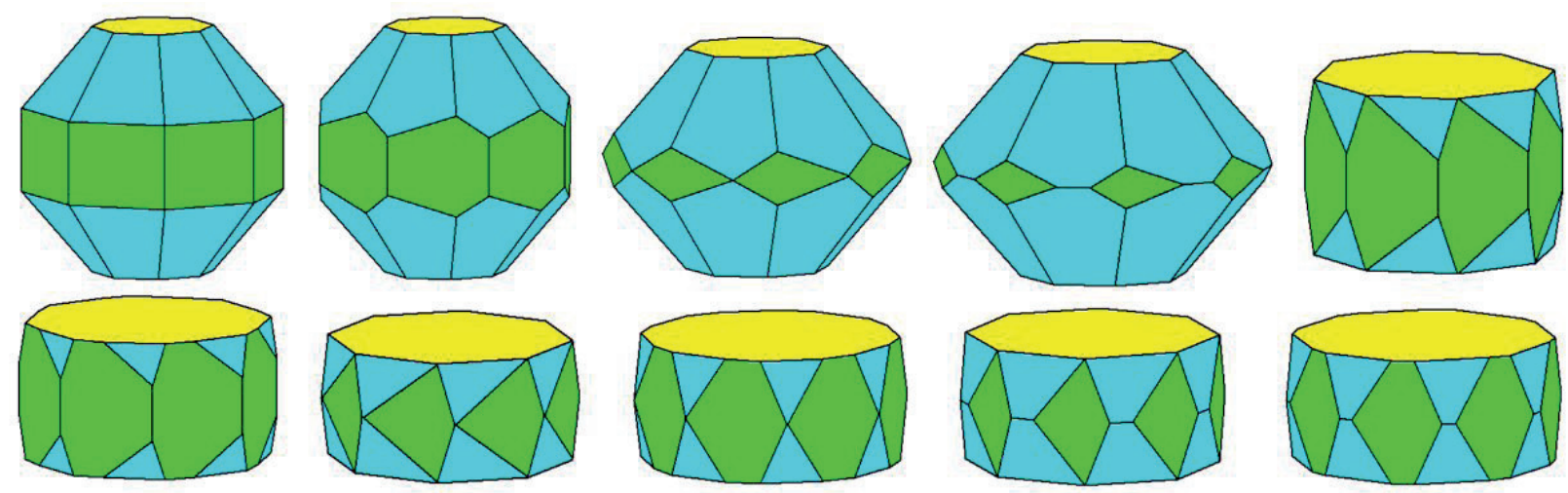

Рис. 5. Все комбинации пинакоида, n-гональной призмы и n-гональной дипирамиды.

Fig. 5. All combinations of pinacoid, n-gonal prizma and n-gonal bipyramid.

Таблица 4. Все комбинации простых форм групп симметрии n/m и -(2n) при нечетном n, порождающие полиэдры с числом граней, меньшим, чем 4n.

Table 4. All combinations of the simple forms for $n / m$ and -(2n) symmetry groups with odd $n$, producing polyhedra with number of faces less than $4 \mathrm{n}$.

\begin{tabular}{c|c|c|c|c} 
№ & пинакоид & $\begin{array}{c}\text { n-гональные } \\
\text { призмы }\end{array}$ & $\begin{array}{c}\text { n-гональные } \\
\text { дипирамиды }\end{array}$ & число граней \\
\hline 1 & & 1 & 1 & $2 \mathrm{n}$ \\
2 & & 1 & $3 \mathrm{n}$ \\
3 & 1 & 1 & 1 & $2 \mathrm{n}+2$ \\
4 & 1 & 1 & 1 & $\mathrm{n}+2$ \\
5 & 1 & 2 & & $3 \mathrm{n}+2$ \\
6 & 1 & & & $2 \mathrm{n}+2$
\end{tabular}

При $\mathrm{n}=3,4$ и 6, используя рисунок 1, получим комбинаторные типы для оставшихся кристаллографических групп симметрии, указанные в (Войтеховский, Степенщиков, 2020, рис. 2). При любом другом $\mathrm{n}$ можно получить простейший комбинаторный тип для любой некристаллографической группы симметрии средней категории. Чтобы закрыть множество всех некристаллографических групп симметрии, осталось рассмотреть две икосаэдрические группы $-3-5 m$ и 235 . Для $-3-5 m$ простейший комбинаторный тип найден среди известного полного многообразия 12-эдров - это додекаэдр (рис. 6 a), а для 432 - одна из простых форм этой группы с 60 гранями общего положения (рис. 6 b). Таким образом, нами получены простейшие комбинаторные типы для всех точечных групп симметрии.
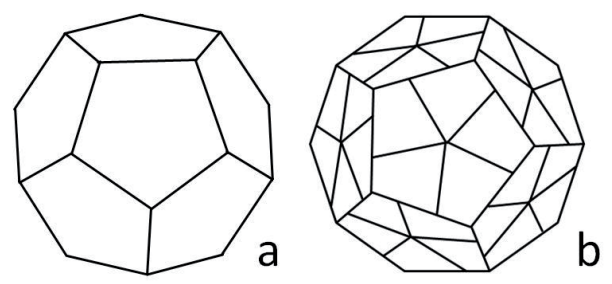

Рис. 6. Простейшие комбинаторные типы выпуклых полиэдров с комбинаторной симметрией -3-5m (a) и 235 (b).

Fig. 6. The simplest combinatorial types of convex polyhedra with combinatorial symmetry $-3-5 m$ (a) and 235 (b).

\section{Литература}

1. Войтеховский Ю.Л., Степенщиков Д.Г. Простейшие комбинаторные типы выпуклых полиэдров с кристаллографическими группами симметрии // Tp. XVI Всерос. Ферсмановской научн. сессии. Апатиты, Геол. ин-т КНЦ РАН, 5-8 апр. 2020 г. Апатиты. Изд-во: КНЦ РАН. 2020. (Наст. сб.) 